\title{
Compact Climbing Robot Rolling on Flexible Magnetic Rollers, for Generator Inspection with the Rotor Still Installed
}

\section{Conference Paper}

Author(s):

Fischer, Wolfgang; Caprari, Gilles; Siegwart, Roland; Moser, Roland

Publication date:

2011

Permanent link:

https://doi.org/10.3929/ethz-a-010025777

Rights / license:

In Copyright - Non-Commercial Use Permitted 


\title{
COMPACT CLIMBING ROBOT ROLLING ON FLEXIBLE MAGNETIC ROLLERS, FOR GENERATOR INSPECTION WITH THE ROTOR STILL INSTALLED
}

\author{
WOLFGANG FISCHER, GILLES CAPRARI, ROLAND SIEGWART \\ Autonomous System Lab, ETH Zürich, \\ Tannenstrasse 3, 8092 Zürich, Switzerland \\ ROLAND MOSER \\ $R \& D$ in Inspection Technologies, ALSTOM Power, \\ Brown Boveri Str. 7, 5401 Baden, Switzerland
}

\begin{abstract}
This paper describes the design and prototype implementation of a compact climbing robot with magnetic adhesion, which is only $8 \mathrm{~mm}$ high and allows for moving in any direction on the stators or rotors of power generators. Thanks to a new locomotion system based on flexible magnetic rollers (= magnetic wheels fixed on motorized flexible shafts; patented technology), the robot is able to span over gaps and non-magnetic zones both in axial and circumferential direction while still being flexible enough for adapting to the large curvature of the rotor/stator $(\sim 0.5 \mathrm{~m}-2 \mathrm{~m})$. With this new design, it is now possible for the first time to access all types of large power generators $(>100 \mathrm{MW})$ with only one device - while still keeping the mechanical complexity at a level which allows for realizing the robot in a robust way and at reasonable cost. After a brief description of the industrial environment where it is supposed to be used (inspection of generator stators with the rotor still installed), this paper describes the basic mechanical concept, pointing to the core innovation and the advantages towards previous designs. The paper concludes with a prototype implementation and some test results; and provides an outlook on future extensions in the final industrial version.
\end{abstract}

\section{Introduction}

This paper describes the design and prototype implementation of a new robotic crawler for the inspection of stators in generators. These generators are installed in every power plant and have to be checked regularly for failures, to assure that the power plant works safe and reliable. As each day of outage can easily cost up to one million Euros, the most important goal for these inspections is to be as fast as possible. Thus, ideally the most time-consuming work step - the removal of the rotor - should be avoided by using inspection devices of very small size. These devices should to be small enough to fit between stator and rotor but still mobile enough to move along the stator geometry that is normally formed out of 
several rows of teeth (Fig. 1, c). In some generators, internal obstacles on the stator (zone-rings) can be found - which require an advanced robot mobility to move around them. In the generators with the most narrow entrance gap, the maximum allowed vehicle height is limited to $9 \mathrm{~mm}$ (Fig. 1, b).

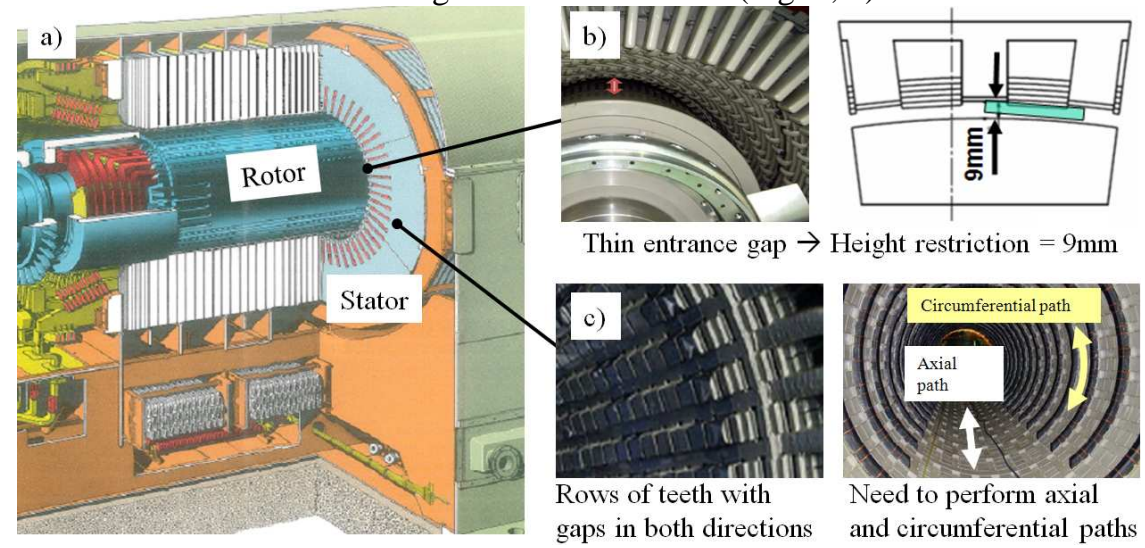

Fig. 1: Sketch of a generator (a), with the entrance geometry that limits the vehicle height (b), and the typical structure of the stator (c) that sometimes contains internal obstacles (zone-rings)

The robot which is presented in this paper is the first one which is able to deal with all these challenges at once, while still keeping a mechanical structure that allows for realizing it in a robust way and at reasonable cost.

The paper is structured is as follows:

Section 2 provides a brief overlook on existing climbing robots for generator inspection and shows their main limitations. The basic locomotion concept of our robot and its advantages towards previous systems is explained in Section 3. This section is followed by the realization of the prototype and the results from the first tests, concluding with an outlook to planned extensions in the final industrial version.

\section{State of the art}

For inspecting power generators, mainly robots with permanent magnets for adhesion and tracks for locomotion have been realized, such as the DIRIS Flex (Fig. 2-a, [1]) or the Siemens FastGen (Fig. 2-b, [2]). Both are too big for passing the most narrow entrance gaps ([1]: 50mm, [2]: $12.5 \mathrm{~mm})$ and cannot move on circumferential paths either. Another competitor product is the GE MAGIC (Fig. 2-c, [3]). This robot achieves axial and circumferential mobility by using three pairs of tracks, with a pneumatic spring in between that allows for spreading between stator and rotor. With this advanced mobility and a height of $12.7 \mathrm{~mm}(0.5$ "), its application scope is already bigger than the two previously 
mentioned robots; however it is not yet able to pass the most narrow entrance gaps of only $9 \mathrm{~mm}$ height. Another recent development is the DIRIS small (Fig. 2-d, [4]), which is a manipulator that pushes a flexible rod into the generator air gap and can move on circumferential paths on the end ring. With this system, it is possible to inspect generators with a $9 \mathrm{~mm}$ entrance gap and theoretically even smaller, but generators with internal obstacles (zone-rings) cannot be accessed.
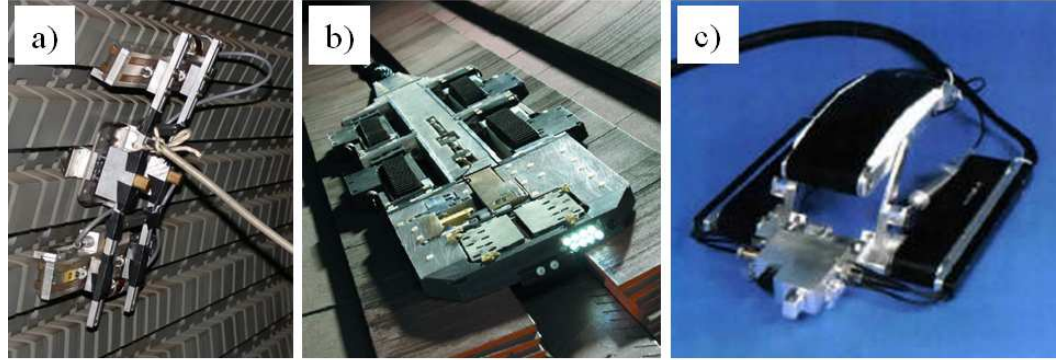

Other mobile robots for generator inspection $\rightarrow$ Height still too large

d)
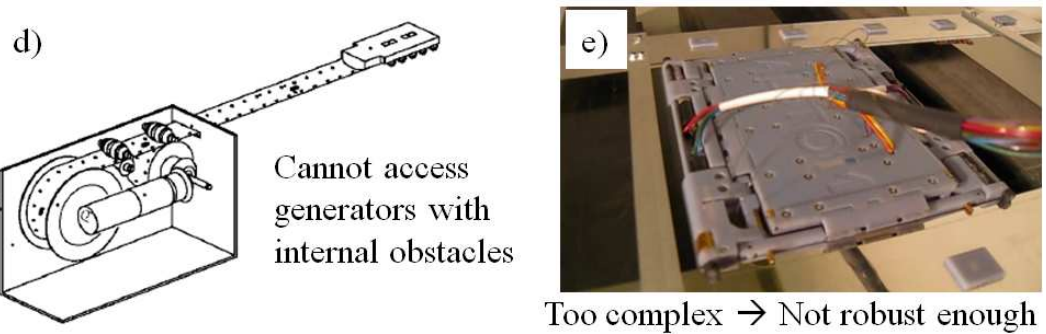

Too complex $\rightarrow$ Not robust enough

Fig. 2: Previous robotic systems for generator inspection and their main limitations: (a) DIRIS Flex [1], (b) Siemens FastGen [2], (c) GE MAGIC [3], (d) DIRIS small [4], (e) Previous design by our research team $[5,6]$

With the goal to achieve the required low height with a mobile robot, a magnetic wheeled climbing robot has been developed by our research team (Fig. 2 -e, $[5,6])$. The significant reduction of height in comparison to the competitor products could be achieved by using several shafts with magnetic wheels for adhesion and locomotion (instead of magnets in the chassis and tracks), and spur gears for transmitting the torque to the wheels. For passing the gaps in circumferential direction, this robot can transfer its adhesion zone to a frame that runs parallel to the wheel unit and thus move to the next row of teeth in a way that is similar to inchworm-locomotion. The first prototype of this robot performed well in the test-environment; however its robustness was not regarded sufficient enough for real industrial application.

For this reason, the main goal for the here presented robot was set to achieve a mobility in the specified environment which is similar to our previous prototype, but with a mechanical structure that is simple, robust and reliable. 


\section{Basic mechanical concept}

For achieving advanced mobility at very small height and complexity, the new concept is based on a combination of advantageous features taken from previously developed robots: Simple differential-drive-steering like in the GE MAGIC [3] (mobility in all directions at reasonable complexity); but magnetic adhesion for avoiding the spring system that spreads towards the other surface (difficult to realize below $9 \mathrm{~mm}$ height, patented by GE).

This combination is realized in a new robot structure. It can be bent at a large radius (approximately $0.5-2 \mathrm{~m}$ ) but is yet stiff enough to span over small gaps or non-magnetic zones (up to $30 \mathrm{~mm}$ ). Motorized magnetic wheels are distributed along most of its area for providing adhesion and traction.

The structure consists of a flexible frame which holds two flexible traction units on both sides and the payload in the center - comparable to the structure of a catamaran-boat (Fig. 3, a). The flexibility of the traction units is achieved by flexible magnetic rollers - magnetic wheels glued to flexible shafts and connected to a gear-motor with spur-gears (Fig. 3, b). With this structure, the robot is able to adapt to any curvature between straight and the radius of the stator, while still being stiff enough for spanning over the gaps between the stator teeth and providing enough adhesion force towards the surface for not slipping - both on axial paths (Fig. 3, c, left) and on circumferential paths (Fig. 3 , c, right).
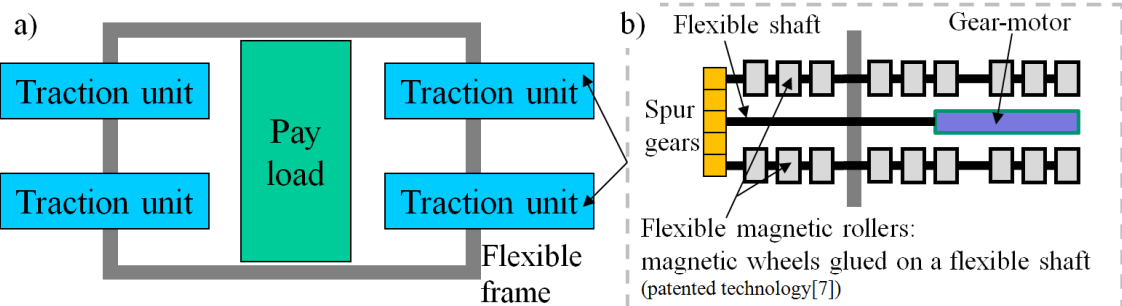

c)
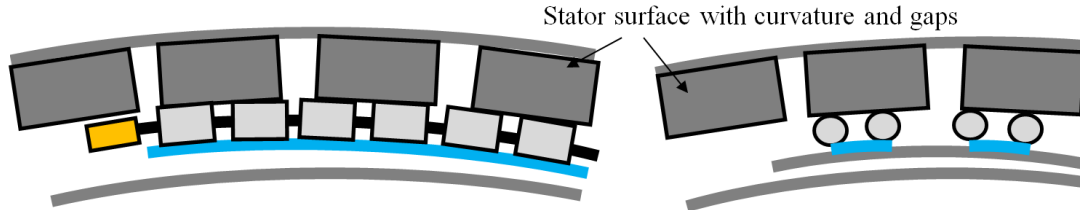

Fig. 3: Basic mechanical concept for the robot: (a) Entire vehicle structure, (b) Detail view of a traction unit with flexible magnetic rollers (patented technology [7], (c) Adaptation of the flexible magnetic roller to a curved ferromagnetic surface with gaps, as it is found in generator stators 
With this innovative vehicle structure, a similar mobility as in the GE MAGIC can be achieved (allows for accessing generators with internal obstacles). Furthermore, the mechanical complexity is even lower, which allowa for significantly reduced height (no need for an actively actuated spring system for spreading anymore). Also in comparison to our own previous system [6] the mechanical complexity is reduced significantly.

\section{Prototype realization}

Based on this concept we realized a prototype for proving its functionality. It achieves a height of only $8 \mathrm{~mm}$, at $150 \mathrm{~mm}$ length, $350 \mathrm{~mm}$ width, and a mass of approximately $200 \mathrm{~g}$ (see Fig. 4).

The frame is realized with a combination of thick parts $(3-5 \mathrm{~mm})$ out of plastics and thin spring-steel bands $(0.05 \ldots 0.3 \mathrm{~mm})$ that are clamped between these plastic parts. By changing the free length and the thickness of these steelbands, the stiffness can be adjusted.

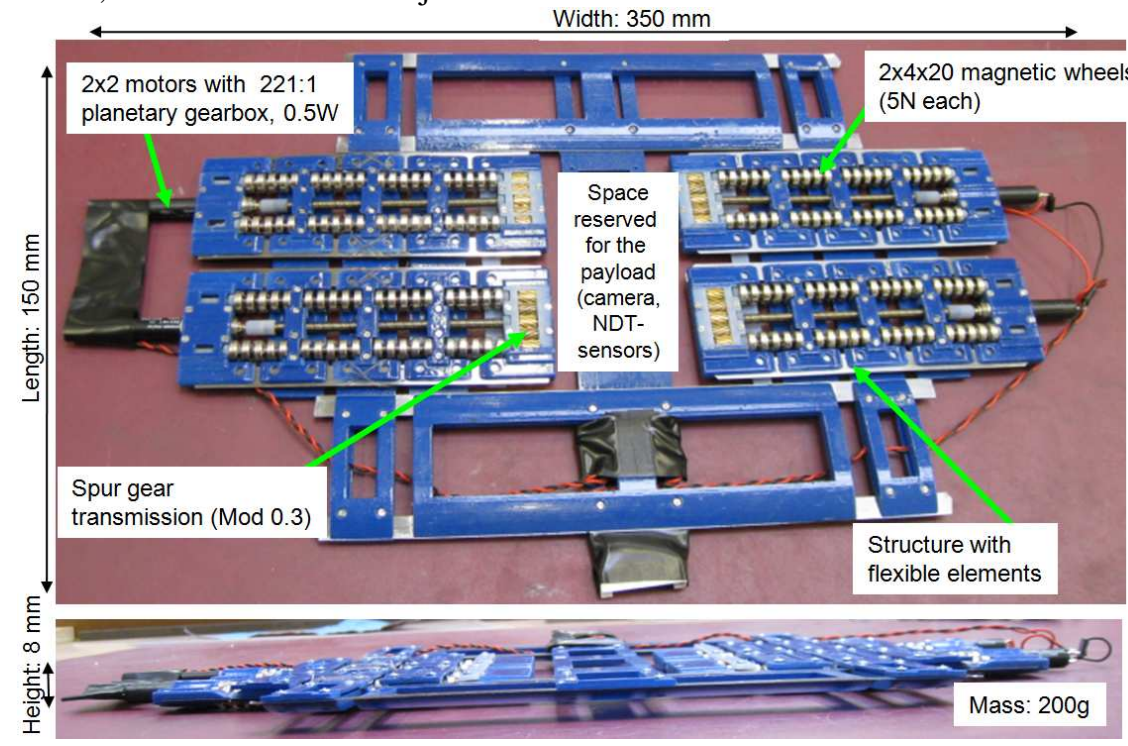

Fig. 4: First test prototype - size, mass and main features

Each traction unit consists of 2 flexible magnetic rollers with 20 ring magnets each $(\varnothing 3-6 \mathrm{~mm}, \mathrm{~b}=2 \mathrm{~mm}, \mathrm{~F}=5 \mathrm{~N})$ that are fixed on an $\varnothing 3 \mathrm{~mm}$ flexible shaft. The torque is provided by $\varnothing 6$ DC-motors with planetary gearboxes (Maxon RE6 with GP6, 4 stages, $\mathrm{i}=221: 1, \mathrm{P}=0.5 \mathrm{~W}$, T_nominal $=30 \mathrm{mNm}$ ) and transmitted to the rollers via a flexible shaft and a spur-gear-transmission $(2 \times 2$ stages without reduction, mod 0.3, 12 teeth, helical shape, brass). 
For increasing the stiffness against torsion and bending along an axis normal to the surface (necessary for optimally transmitting the motor torque to the wheels) while still keeping the flexibility to bend around an axis parallel to the driving direction (curvature-adaptation); the structure of the traction units is formed similar to the sandwich-design in skis and snowboards: stiff sections combined with flexible ones, and the intersection lines parallel to the driving direction. Similar to the frame, the stiff sections are manufactured out of relatively thick plastic parts, while the flexible ones are realized with thin springsteel bands that are clamped between the stiff sections.

For carrying the planned payload (camera + sensors for non-destructive testing, approximately $100 \mathrm{~g}$ of total mass), a section of approximately $100 \times 50 \mathrm{~mm}$ is foreseen in the middle of the frame. By changing the geometry of the frame, this space can be increased if necessary. Also a $3^{\text {rd }}$ or $4^{\text {th }}$ pair of traction units could be added, if heavy payloads need to be carried.

In this first prototype, the robot is still manually controlled by the operator using 2 bi-directional switches that apply either positive or negative voltage on all traction units of each side.

\section{Test results}

After preliminary tests at ETH Zürich, the robot was then tested on real generators in the ALSTOM-factory in Birr (Switzerland) - on a rotor with $2 \mathrm{~m}$ diameter (Fig. 5, a), an unpainted stator with approximately $1.5 \mathrm{~m}$ diameter, and gaps up to $30 \mathrm{~mm}$ (Fig. 5, b) and a painted stator with approximately $2 \mathrm{~m}$ diameter, gaps up to $30 \mathrm{~mm}$, and paint drops of up to $2 \mathrm{~mm}$ height (Fig. 5, c).
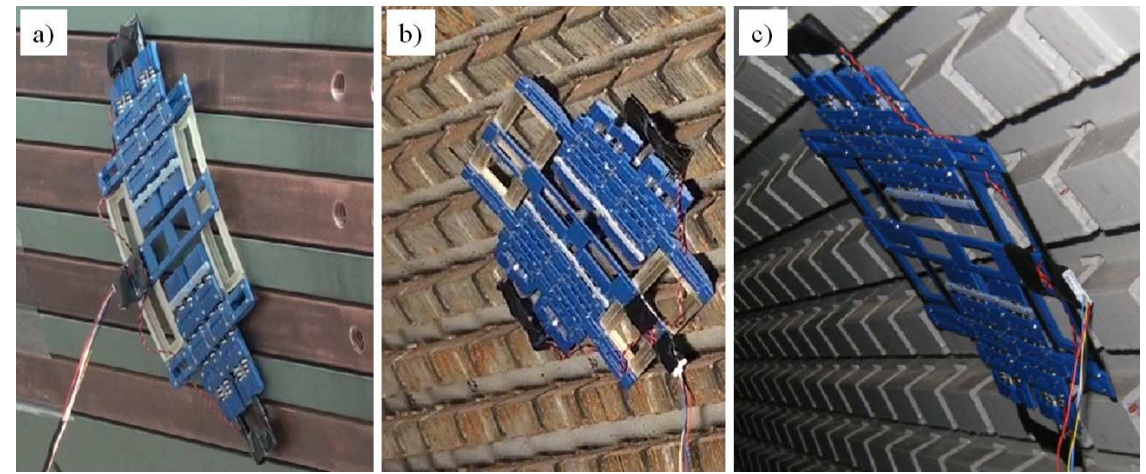

Fig. 5: Tests in real generators: (a) rotor, (b) unpainted stator of small diameter, (c) stator with paint drops up to $2 \mathrm{~mm}$ height 
In all these environments, the prototype was stable in all possible inclinations in respect to gravity and was able to carry twice the specified payload (200g - more was not tested, as there is anyway no additional space in this narrow environment). Regarding its mobility, all zones in the rotor and in the unpainted stator could be accessed without problems. Only on the painted stator the robot sometimes got blocked on some of the bigger paint drops (approximately $2 \mathrm{~mm}$ height; driving back again was still possible). This limitation has to be mentioned, but should not be severe, as on these painted generators it is possible to place the robot either on the stator- or on the rotorsurface (using a special insertion-stick). When driving on the rotor, the robot cannot move completely to the stator, but is able to access it with its tools by using a small manipulator-arm that covers the distance $(20-50 \mathrm{~mm}$ inside the generator). The detailed design of such a manipulator-arm is planned for the next phase of this project.

The maximum driving speed was measured to approximately $1.5 \mathrm{~m} / \mathrm{min}$, which is similar to our previous prototypes that use the same gear-motors [5]. With these speed values, the necessary time for scanning a generator of typical size (6m length, 60 rows of teeth) can be calculated to approximately 4-8 hours. This time is significantly lower than the necessary time for removing the rotor (0.5-1 day) and does not bring the risk of damaging it. For further increasing this speed at similar payload-capability, bigger gear-motors than the currently used ones $(\mathrm{D}=6 \mathrm{~mm}, \mathrm{P}=0.5 \mathrm{~W})$ would be necessary. Such a bigger version of the robot could be an option for larger generators where the entrance-gap is less narrow.

The inclination of the path (= going straight, upwards or downwards) only plays a minor effect $(\sim 10 \%)$ on the driving-speed, which is almost proportional to the input-voltage on the motors. For this reason, an almost constant speed can already be achieved without using encoders and motor-controllers.
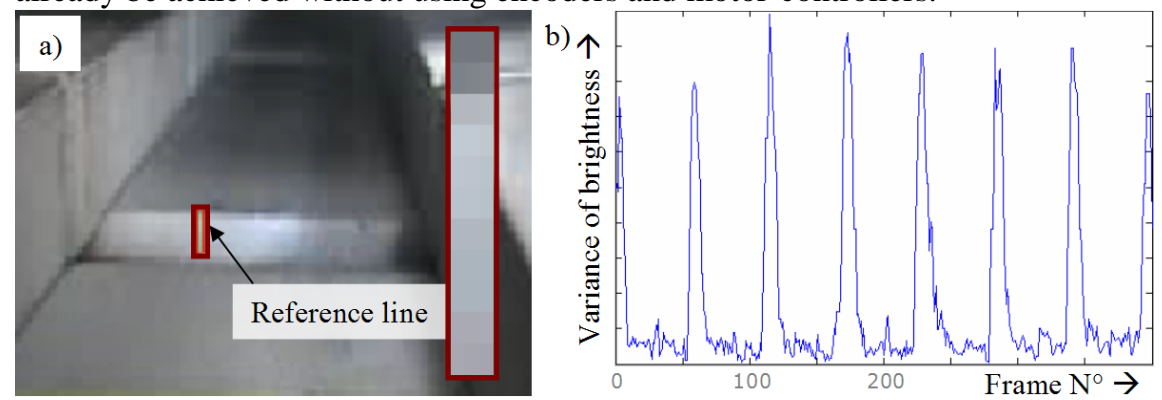

Fig. 6: Detection of the edges directly from the camera-image (a) Typical camera-image with a reference line which is 10 pixels long, (b) Variance of the color-value along the reference line for 400 frames in a movie where the robot is rolling in axial direction at constant speed. The peaks in the graph clearly indicate when the camera is above the edge of a stator tooth. 
For the localization of the robot, it is planned to avoid additional sensors that increase its complexity and only use the image of the onboard-camera. These images are usually in very good quality, as there is no other light except the LEDs integrated in the camera. Furthermore, the environment is highly structured and contains many edges that are easily detectable with standard lineextraction-algorithms. Preliminary tests with a very simple program for detecting the edges of a typical camera-image-stream (still taken with the DIRIS small [4]) already showed quite promising results (see Fig. 6).

Ongoing work now consists in developing a program that implements an adapted line-following-algorithm for keeping a straight path, counts all edges for localizing the robot's position and automatically generates a high-resolutionimage of the generator where the operator can see potential failures much faster than by looking at the camera-stream alone.

\section{Conclusion and outlook to future work}

In this paper, we have presented the design and prototype implementation of a compact climbing robot with magnetic adhesion, which is only $8 \mathrm{~mm}$ high and allows for moving in any direction on the stators or rotors of power generators at a relatively low system complexity, thanks to a new locomotion system based on flexible magnetic rollers. The robot was tested in real environment, where it performed well and could clearly show its advantages towards previous systems.

With this new design, it is now possible for the first time to access all types of large power generators $(>100 \mathrm{MW})$ with only one device - while still keeping the mechanical complexity at a reasonable level that allows for realizing the robot in a robust way and at reasonable cost. This ability will make it a valuable tool for most type of generator inspections, and help to significantly decrease the outage-time of power plants during inspection. For this reason, the core innovation was also patented.

Ongoing work towards the industrialization of this robot mainly includes the optimization of the structure for more robustness (Al and stainless steel instead of plastics) and the implementation of the control-program. 


\section{Acknowledgments}

This work was supported by grant KTI 8435.1 EPRP-IW of the Swiss "Bundesamt für Berufsbildung und Technologie" and ALSTOM Power, Baden, Switzerland. We also want to thank all people at ALSTOM Power and ALSTOM Inspection-Robotics who supported us during the design-phase and the tests, especially Niklaus Hugi, Reinhard Fischer, Markus Wiesendanger and Igor Thommen.

\section{References}

1. R. Moser, B. Mark; "Automated Robotic Inspection of Large Generator Stators"; in Proceedings of the IEEE/ASME International Conference on Advanced Intelligent Mechatronics; September 4-7, 2007, ETH Zurich, Switzerland.

2. D. Schoeler, P.O. Miranda; "Improved Unit Reliability \& Availability Through Optimised Predictive Maintenance"; Siemens AG, Power Generation; 2003.

3. D.T. Roney, R. J. Zawoyski; "Generator In-Situ Inspections"; GE Power Systems, found on http://www.gepower.com/prod_serv/serv_for/generators/en/testing_insp/ndt.htm

4. R. Moser, N. Hugi, M. Bernhard, P. Isler, S. Honold, J. Erni, "Vorrichtung zur Inspektion eines Spaltes", Patent EP 2110678 A1, Alstom Technology Ltd., 14.4.2008.

5. W. Fischer, G. Caprari, R. Siegwart, R. Moser, "Robotic Crawler for Inspecting Generators with Very Narrow Air Gaps", in Proceedings of IEEE-ICM 2009, IEEE-IES International Conference on Mechatronics, Malaga, Spain, April 2009.

6. W. Fischer, G. Caprari, R. Siegwart, R. Moser, "Locomotion System for a Mobile Robot on Magnetic Wheels With Both Axial and Circumferential Mobility but only 8mm Height for Generator Inspection With the Rotor Still Installed", accepted for publication at Transactions on Industrial Electronics, 2011, not published yet.

7. Markus Wiesendanger, Wolfgang Fischer, "DEVICE FOR INSPECTION OF GENERATOR AIR GAPS”, Patent US 61/296,199, ALSTOM Technology Ltd., Filing Date: 19.01.2010.

8. W. Fischer, G. Caprari, R. Siegwart, I. Thommen, W. Zesch, R. Moser, " Foldable magnetic wheeled climbing robot for the inspection of gas turbines and similar environments with very narrow access holes", Industrial Robot, Vol. 37, Issue 3, pp. 244-249, 2010. 